\title{
Using Isotemporal Analyses to Examine the Relationships Between Daytime Activities and Cancer Recurrence Biomarkers in Breast Cancer Survivors
}

\author{
Kelsie M. Full, Eileen Johnson, Michelle Takemoto, Sheri J. Hartman, Jacqueline Kerr, Loki Natarajan, \\ Ruth E. Patterson, and Dorothy D. Sears
}

\begin{abstract}
Background: For breast cancer survivors, moderate to vigorous physical activity (MVPA) is associated with improved survival. Less is known about the interrelationships of daytime activities (sedentary behavior [SB], light-intensity physical activity, and MVPA) and associations with survivors' health outcomes. This study will use isotemporal substitution to explore reallocations of time spent in daytime activities and associations with cancer recurrence biomarkers. Methods: Breast cancer survivors $(\mathrm{N}=333$; mean age 63 y) wore accelerometers and provided fasting blood samples. Linear regression models estimated the associations between daytime activities and cancer recurrence biomarkers. Isotemporal substitution models estimated cross-sectional associations with biomarkers when time was reallocated from of one activity to another. Models were adjusted for wear time, demographics, lifestyle factors, and medical conditions. Results: MVPA was significantly associated with lower insulin, C-reactive protein, homeostatic model assessment of insulin resistance, and glucose, and higher sex hormone-binding globulin (all $P<.05$ ). Light-intensity physical activity and SB were associated with insulin and homeostatic model assessment of insulin resistance (both $P<.05$ ). Reallocating 18 minutes of SB to MVPA resulted in significant beneficial associations with insulin $(-9.3 \%)$, homeostatic model assessment of insulin resistance $(-10.8 \%)$, glucose $(-1.7 \%)$, and sex hormone-binding globulin (7.7\%). There were no significant associations when 79 minutes of SB were shifted to light-intensity physical activity. Conclusions: Results illuminate the possible benefits for breast cancer survivors of replacing time spent in SB with MVPA.
\end{abstract}

Keywords: lifestyle behaviors, accelerometers, survivorship, insulin resistance

Breast cancer remains the most common cancer among women. ${ }^{1-3}$ Currently, in the United States, an estimated 3.5 million women are living with a history of breast cancer. ${ }^{2}$ Due to earlier diagnosis and advances in treatment methodologies, the likelihood of survival after a breast cancer diagnosis continues to increase. ${ }^{2}$ Survivors face a new set of challenges as they remain at risk for cancer recurrence and mortality. ${ }^{4,5}$

Several breast cancer risk-related biomarkers have been found to be significantly associated with poor lifestyle factors such as reduced physical activity (PA) and elevated body mass index (BMI). These biomarkers are related to glucoregulation, chronic inflammation, and endogenous sex hormones and are thought to be mechanistic drivers of cancer progression. ${ }^{6-10}$ Notably, elevated fasting insulin, estradiol, and C-reactive protein (CRP) are associated with an approximate 2-fold increased risk of cancer risk or recurrence. ${ }^{11-16}$

For survivors, many lifestyle behavior treatment plans focus on increasing minutes of daily PA to reduce risk of cancer recurrence. ${ }^{5}$ Meta-analyses and reviews provide consistent evidence showing that daily PA, and more specifically moderate to vigorous physical activity (MVPA), is linked to better breast cancer

Full is with the Division of Epidemiology and Community Health, University of Minnesota, Minneapolis, MN, USA. Full, Takemoto, Hartman, Kerr, Natarajan, Patterson, and Sears are with the Department of Family Medicine and Public Health, University of California, San Diego, La Jolla, CA, USA. Johnson is with the Division of Epidemiology, School of Public Health, University of California Berkeley, Berkeley, CA, USA. Hartman, Natarajan, Patterson, and Sears are also with the UC San Diego Moores Cancer Center, University of California, San Diego, La Jolla, CA, USA. Sears is also with the College of Health Solutions, Arizona State University, Phoenix, AZ, USA. Full (fullx003@umn.edu) is corresponding author. survival outcomes. ${ }^{17,18}$ A meta-analysis by Beasley et al ${ }^{19}$ concluded that survivors who had at least 10 MET hours of PA per week (ie, $180 \mathrm{~min}$ of MVPA) had 27\% lower mortality than survivors with lower levels of MVPA. Despite the suggested benefits of PA, only $32 \%$ of breast cancer survivors are meeting current PA guidelines of 150 minutes of weekly MVPA. ${ }^{20,21}$

Besides MVPA, there is limited evidence on how the other activities that make up the waking hours of the day, including sedentary behavior (SB) and light-intensity physical activity (LIPA), are related to breast cancer recurrence. Independent of the benefits of PA, prolonged sitting is associated with increased risk of cancer. ${ }^{22-24}$ We have recently shown in breast cancer survivors that a greater number of sit-to-stand transitions each day (indicative of interrupted sitting patterns) is significantly associated with reduced fasting insulin, insulin resistance, and CRP. ${ }^{25}$ Furthermore, engaging in LIPA has shown to have beneficial associations with glucoregulatory markers, including blood glucose and insulin, after adjusting for minutes of MVPA. ${ }^{26,27}$ Therefore, reducing SB or increasing LIPA may be feasible alternatives for breast cancer survivors who are currently not meeting MVPA guidelines.

The 24-hour activity behaviors are relative, meaning reducing time spent in one activity directly corresponds to increasing time spent in another activity. Isotemporal substitution modeling techniques $^{28}$ are used to explore how the interrelationships of daily activities may be associated with health, and to estimate the associations if a fixed amount of time spent in one activity is reallocated to another activity. In Figure 1, we provide a conceptual example of daytime activity behavior substitution. During this day, a 30-minute decrease in SB could be the result of either reallocating an additional 30 minutes to LIPA or an additional 30 minutes to MVPA. The 


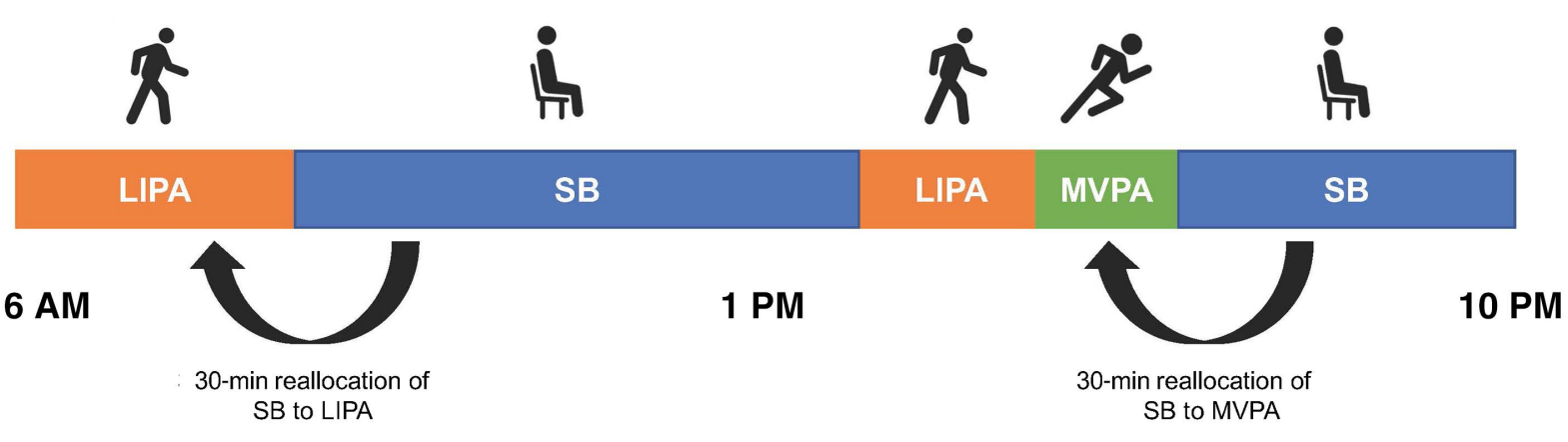

Figure 1 - A conceptual example of activity behavior substitution. LIPA indicates light-intensity physical activity; MVPA, moderate to vigorous physical activity; SB, sedentary behavior.

isotemporal substitution approach allows us to examine the hypothetical associations with health outcomes when time spent in one activity is reallocated to another activity. In contrast to a behavioral intervention, which may not achieve sufficient reductions in behaviors to understand the impact on risk, this statistical modeling technique allows us to estimate the potential impact of meaningful behavioral substitution. Recent isotemporal studies in breast cancer survivor populations suggest it is worthwhile to move beyond examining daily activities independently and start to explore how time spent in activities throughout the day is associated to health. ${ }^{29,30}$

The primary aim of this study is to use accelerometer data to construct isotemporal substitution models to assess the associations to cancer recurrence biomarkers of reallocating time spent in SB, LIPA, and MVPA in a sample of postmenopausal breast cancer survivors. The goal of these analyses is to explore how the reallocation of time spent in daytime activities may be beneficial to markers of breast cancer recurrence. This study may provide insight on current cancer lifestyle treatment guidelines and potentially highlight opportunities for the development of novel survivorship interventions.

\section{Methods}

\section{Sample and Study Design}

The current analyses use baseline data from participants enrolled in The Reach for Health study. ${ }^{31}$ To be eligible for participation in the study, women must be overweight or obese with a history of earlystage postmenopausal breast cancer. Women were excluded from the study if they had diabetes, kidney failure or liver impairment, or were diagnosed with an additional primary or recurrent invasive cancer within the last 10 years. Data were collected between August 2011 and May 2015. The UC San Diego Institutional Review Board approved all study procedures, and all participants provided written informed consent.

A full description of the study protocol has been previously published. ${ }^{31}$ In brief, women who appeared to be eligible for the study based on a telephone interview were invited to a screening visit to confirm eligibility. After completion of the screening visit, participants completed a questionnaire, physical measurements, and were given a hip-worn accelerometer. Participants were asked to wear the device during waking hours for 7 days and return at the follow-up baseline visit.

All 333 of the women participating in the Reach for Health study were included in this analysis with valid accelerometer data, a completed study questionnaire, and fasting biomarker results.

\section{Daytime Activities}

Daytime activities were assessed with a hip-worn triaxial accelerometer (ActiGraph GT3X accelerometer; ActiGraph LLC, Pensacola, FL). Participants were instructed to wear the device during waking hours and remove the device for bathing, swimming, and sleeping. Participants received oral and written instructions on how to properly wear the device and received 2 reminder phone calls from study staff during the 7-day wear period. Participants returned the accelerometers by mail at the end of the wear period. Raw accelerometer data was recorded at $30 \mathrm{~Hz}$.

After devices were returned, data were downloaded using ActiLife software (version 6.3.4; ActiGraph) and screened for device wear time using the validated Choi wear time criteria. ${ }^{32}$ According to the criteria, 90 consecutive minutes of zero counts were classified as nonwear, and a 2-minute spike tolerance and a 30-minute small window were applied to remove artifactual movement. ${ }^{32}$ To be considered valid, a day must include at least 600 minutes $(10 \mathrm{~h})$ of wear. Sufficient wear time was classified as 5 days with $\geq 600$ minutes or $>3000$ minutes over a 4 -day period. Participants who did not meet the minimum criteria were asked to rewear the device. The GT3X ActiGraph device (ActiGraph), worn on the hip, has been used previously to assess PA and SB in older adult samples. ${ }^{33-36}$

Daytime activity behaviors were classified as previously described.$^{37}$ Each minute of wear time was classified according to intensity (counts per minute) using established cut points as follows: SB (0-99), LIPA (100-1951), and MVPA (1952+). ${ }^{38}$ For each valid day, the estimated total time spent in activities (SB, LIPA, and MVPA) was approximated by summing the total number of minutes classified in each activity intensity. Day-level estimates of minutes spent in SB, LIPA, and MVPA were averaged across all valid days per participant to create average time spent in SB, LIPA, and MVPA. An estimate of average total PA was calculated by summing the minutes of LIPA and MVPA.

\section{Cancer Recurrence Biomarkers}

From overnight fasting blood samples collected at the clinic visit, buffy coat and red blood cell samples were obtained. Plasma and serum were used to measure insulin, glucose, CRP (plasma), bioavailable sex hormones (estradiol and testosterone), and sex hormone-binding globulin (SHBG; serum), as described previously. ${ }^{39}$ Overnight fasting is the standard method used to clinically control for dietary effects on these biomarkers. Intraplate and 
interplate coefficients of variance, respectively, were $3.5 \%$ and $6.5 \%$ for insulin, $2.1 \%$ and $3.2 \%$ for glucose, and $8.4 \%$ and $18.0 \%$ for CRP, measured at UC San Diego as reported previously. Serum estradiol, testosterone, and SHBG were measured at the Reproductive Endocrine Research Laboratory at the University of Southern California (Director: Frank Z. Stancyzk). Assay sensitivities for testosterone and estradiol were $1.5 \mathrm{ng} / \mathrm{dL}$ and $2 \mathrm{pg} / \mathrm{mL}$, respectively; values for all participants were above the assay sensitivities. Intraassay and interassay coefficients of variance ranged from $3 \%$ to $6 \%$ and $9 \%$ to $12 \%$, at low and high levels, respectively, in quality control samples. SHBG assay sensitivity was $0.1 \mathrm{nmol} / \mathrm{L}$, and the intraassay coefficients of variance was $7.0 \%$. Blood was drawn immediately following the accelerometer wear time period and, thus, is reflective of the physiological state associated with the daytime activity measured.

\section{Covariates}

At the screening visit, participants self-reported demographic information including age, race (categorized as white or European American, black or African American, or other), and level of education (categorized as high school or less, some college, college graduate, or graduate school). Additional information about lifestyle behaviors and health status collected from the baseline questionnaire included self-reported smoking status (never, former, and current), alcohol consumption in the past 3 months (yes or no), and medical conditions including high blood pressure and high cholesterol. Age at breast cancer diagnosis, cancer stage and medication being taken at study enrollment (including aromatase Inhibitors) were obtained through medical chart reviews. BMI (measured in kilogram per square meter) was calculated from height and weight measures.

\section{Statistical Analysis}

Average minutes of SB, LIPA, and MVPA were included in a series of regression models to examine the associations with each cancer recurrence biomarker. Prior to analysis, values for the biomarkers were log transformed due to their nonnormal distribution and daytime activity variables were summed to create a total wear time variable.

Single activity variable linear regression models estimated the association for each of the activities and the cancer recurrence biomarkers. Adjusted linear regression models were run for each biomarker and SB, LIPA, and MVPA individually. Models adjusted for total wear time and multiple covariates, but not for the other daytime activities. Models included adjustment for wear time, age, race, education, smoking status, alcohol consumption, age at diagnosis, cancer stage, aromatase inhibitor use, high blood pressure, and high cholesterol.

The associations between daytime activity and the biomarkers of interest could be influenced by medications used to regulate medical conditions such as high blood pressure and high cholesterol. To test the extent to which associations may vary by medical conditions, interactions were tested for high blood pressure (high blood pressure $\times$ activity) and high cholesterol (high cholesterol $\times$ activity) in the single-variable models. In additional sensitivity analyses, we ran the single activity variable models with and without adjusting for BMI.

When significant associations were observed in the single activity variable models, isotemporal substitution models were performed on that biomarker. Isotemporal substitution models estimated the association with the cancer biomarkers of reallocating time from one activity to another activity. The substitution models for each biomarker included a variable for each daytime activity besides the activity of interest, the total wear time variable, and the aforementioned covariates. For example, to test the reallocation of time from SB, both LIPA and MVPA variables would be included in the model with the total wear time variable and covariates. Including a total wear time variable in the model holds time constant and allows for the interpretation of the cross-sectional associations of biomarker levels observed with reducing the mean time spent in one activity by equivalently increasing the mean time spent in another activity. Results are reported for $1 \mathrm{SD}$ unit substitution of the activity of interest.

As data are cross-sectional, it is important to note that the resulting coefficients cannot be interpreted as causal effects, but as estimated associations from a modeled reallocation of a fixed amount of time. All analyses were performed using $\mathrm{R}$ statistical software (version 3.1.1). ${ }^{40}$

\section{Results}

\section{Sample Characteristics}

Characteristics of the 333 postmenopausal breast cancer survivors according to total PA level are presented in Table 1 . The mean age of participants was $62.6(6.9)$ years, and $83.2 \%$ of the women in the study sample were Non-Hispanic white. Approximately $45 \%$ of the women were former or current smokers and almost $80 \%$ had consumed alcohol in the past 3 months. The average BMI of participants was $31.1(5.0) \mathrm{kg} / \mathrm{m}^{2}$, and approximately $50 \%$ of women reported having high blood pressure and $49 \%$ reported having high cholesterol.

Study participants wore the accelerometer for approximately 829 minutes per day (almost $14 \mathrm{~h}$ ) across the 7-day period. On average, participants in the sample recorded 19.9 minutes per day of MVPA $( \pm 17.9), 300.9$ minutes or approximately 5 hours of LIPA $( \pm 79.0 \mathrm{~min})$, and 508 minutes or approximately 8.5 hours per day of SB $( \pm 92.1 \mathrm{~min})$.

Women in the lowest tertile of total daily PA (T1) were older, were more likely to be white or black than other race, had the highest BMI, and often had more unfavorable cancer recurrence biomarker levels compared with those in the higher tertiles of total PA.

\section{Associations of Daytime Activities With Fasting Levels of Cancer Recurrence Biomarkers}

Time spent in the daytime activities was significantly associated with several cancer recurrence biomarkers. The results from the single activity variable models are presented in Table 2. After adjustment for wear time, sociodemographic characteristics, lifestyle behaviors, and health status indicators, MVPA was significantly associated with lower values of insulin $(B=-2.01$, $P<.001)$, CRP $(B=-1.18, P=.04)$, homeostatic model assessment of insulin resistance (HOMA-IR; $B=-2.14, P<.001$ ), and glucose $(B=-1.43, P=.01)$, and higher values of SHBG $(B=1.45, P<.01)$. Similarly, LIPA was significantly associated with lower values of insulin $(B=-1.17, P=.04)$ and HOMA-IR $(B=-1.10, P=.05)$. SB was significantly associated with higher values of insulin $(B=1.71, P<.01)$ and HOMA-IR $(B=1.67$, $P<.01)$. None of the daytime activities were significantly related 
Table 1 Characteristics of Breast Cancer Survivors According to Tertiles of Total PA $(\mathrm{N}=333)$

\begin{tabular}{|c|c|c|c|c|}
\hline & \multirow[b]{3}{*}{ Overall } & \multicolumn{3}{|c|}{ Total PA, min/d } \\
\hline & & T1 & T2 & T3 \\
\hline & & $<280.2$ & 280.2-345.2 & $>345.2$ \\
\hline Age, mean (SD), y & $62.6(6.9)$ & $63.9(7.6)$ & $62.6(6.3)$ & $61.2(6.6)$ \\
\hline \multicolumn{5}{|l|}{ Race, n (\%) } \\
\hline White or European American & $277(83.2)$ & $94(85.5)$ & $91(82.7)$ & $92(81.4)$ \\
\hline Black or African American & $12(3.6)$ & $5(4.5)$ & $3(2.7)$ & $4(3.5)$ \\
\hline Other race & $44(13.2)$ & $11(10.0)$ & $16(14.5)$ & $17(15.0)$ \\
\hline \multicolumn{5}{|l|}{ Education, n (\%) } \\
\hline High school or less & $30(9.0)$ & $9(8.2)$ & $8(7.3)$ & $13(11.5)$ \\
\hline Some college & $132(39.6)$ & $48(43.6)$ & $47(42.7)$ & $37(32.7)$ \\
\hline College graduate & $67(20.1)$ & $23(20.9)$ & $20(18.2)$ & $24(21.2)$ \\
\hline Graduate school & $104(31.2)$ & $30(27.3)$ & $35(31.8)$ & $39(34.5)$ \\
\hline \multicolumn{5}{|l|}{ Smoking status, n (\%) } \\
\hline Never & $184(55.3)$ & $65(59.1)$ & $55(50.0)$ & $64(56.6)$ \\
\hline Former & $143(42.9)$ & $44(40.0)$ & $54(49.1)$ & $45(39.8)$ \\
\hline Current & $6(1.8)$ & $1(0.9)$ & $1(0.9)$ & $4(3.5)$ \\
\hline Consumed alcohol in past $3 \mathrm{mo}, \mathrm{n}(\%)$ & $266(79.9)$ & $86(78.2)$ & $88(80.0)$ & $92(81.4)$ \\
\hline Age at diagnosis, mean (SD), y & $59.9(6.9)$ & $61.3(7.5)$ & $59.8(6.5)$ & $58.8(6.6)$ \\
\hline \multicolumn{5}{|l|}{ Cancer stage, $\mathrm{n}(\%)$} \\
\hline Stage I & $161(48.4)$ & $55(50.0)$ & $49(44.5)$ & $57(50.4)$ \\
\hline Stage II & $116(34.8)$ & $35(31.8)$ & $45(40.9)$ & $36(31.9)$ \\
\hline Stage III & $56(16.8)$ & $20(18.2)$ & $16(14.5)$ & $20(17.7)$ \\
\hline Aromatase inhibitor use at study enrollment, n (\%) & $190(57.0)$ & $65(59.1)$ & $60(54.5)$ & $65(57.5)$ \\
\hline BMI, mean (SD), $\mathrm{kg} / \mathrm{m}^{2}$ & $31.1(5.0)$ & $32.3(5.7)$ & $30.8(4.4)$ & $30.2(4.5)$ \\
\hline \multicolumn{5}{|l|}{ Medical conditions, $\mathrm{n}(\%)$} \\
\hline High blood pressure & $166(49.8)$ & $55(50.0)$ & $60(54.5)$ & $51(45.1)$ \\
\hline High cholesterol & $164(49.2)$ & $51(46.4)$ & $58(52.7)$ & $55(48.7)$ \\
\hline \multicolumn{5}{|l|}{ Activity behaviors, mean (SD), min } \\
\hline Wear time & $828.8(72.6)$ & $801.6(71.0)$ & $828.7(71.6)$ & $855.6(65.7)$ \\
\hline MVPA & $19.9(17.9)$ & $12.7(12.6)$ & $17.3(14.6)$ & $29.4(21.1)$ \\
\hline LIPA & $300.9(79.0)$ & $222.0(38.4)$ & $295.0(21.1)$ & $383.4(60.4)$ \\
\hline SB & $508.0(92.1)$ & $566.8(76.1)$ & $516.4(72.9)$ & $442.7(81.4)$ \\
\hline \multicolumn{5}{|l|}{ Fasting cancer recurrence biomarkers, mean (SD) } \\
\hline Insulin, $\mathrm{pg} / \mathrm{mL}$ & $551.7(324.1)$ & $647.0(399.8)$ & $544.7(309.2)$ & $465.9(215.4)$ \\
\hline C-reactive protein, $\mathrm{mg} / \mathrm{L}$ & $5.9(8.3)$ & $7.1(8.9)$ & $6.4(10.5)$ & $4.2(4.3)$ \\
\hline HOMA-IR & $3.4(2.3)$ & $4.2(3.0)$ & $3.3(2.1)$ & $2.8(1.3)$ \\
\hline Glucose, mg/dL & $103.1(13.8)$ & $105.0(17.5)$ & $101.6(10.1)$ & $102.7(12.8)$ \\
\hline SHBG, nmol/L & $51.1(29.5)$ & $49.0(29.9)$ & $52.7(29.9)$ & $51.4(28.6)$ \\
\hline Bioavailable estradiol, $\mathrm{pg} / \mathrm{mL}$ & $4.1(3.9)$ & $4.7(4.7)$ & $4.0(4.1)$ & $3.5(2.6)$ \\
\hline Bioavailable testosterone, $\mathrm{ng} / \mathrm{dL}$ & $13.3(8.3)$ & $14.8(11.4)$ & $12.7(6.3)$ & $12.4(5.9)$ \\
\hline
\end{tabular}

Abbreviations: BMI, body mass index; HOMA-IR, Homeostatic Model Assessment of Insulin Resistance; LIPA, light-intensity physical activity; MVPA, moderate to vigorous physical activity; PA, physical activity; SB, sedentary behavior; SHBG, sex hormone-binding globulin.

to bioavailable estradiol or bioavailable testosterone in the singlevariable models.

There were no statistically significant interactions between daytime activities and high blood pressure or high cholesterol status on any of the cancer recurrence biomarkers. Although all women were overweight or obese, BMI varied significantly across total PA tertiles. Results of the single activity models with additional adjustment for BMI are presented in Supplemental Table 1 (available online).

\section{Isotemporal Substitution Models: Reallocation of Time Spent in Daytime Activity}

The results of the isotemporal substitution models are displayed in Figure 2. Reallocating 79 minutes of SB to LIPA was beneficially associated with lower values of insulin (estimated percentage change: $-4.6 \%$; $95 \%$ confidence interval [CI], -9.8 to 1.0$), \mathrm{CRP}$ $(-7.6 \% ;-18.7$ to 5.2$)$, and HOMA-IR ( $-4.5 \% ;-10.2$ to 1.6$)$, but none of these associations were significant. 


\section{Table 2 Single Activity Variable Models for Daytime Activities and Fasting Levels of Cancer Recurrence Biomarkers $(\mathrm{N}=333)$}

\begin{tabular}{|c|c|c|c|}
\hline Cancer recurrence biomarkers & $B^{a}$ & $\mathrm{SE}^{\mathrm{a}}$ & $P$ value \\
\hline \multicolumn{4}{|l|}{ Insulin, $\mathrm{pg} / \mathrm{mL}$} \\
\hline SB & 1.71 & 0.62 & $<.01$ \\
\hline LIPA & -1.17 & 0.56 & .04 \\
\hline MVPA & -2.01 & 0.55 & $<.001$ \\
\hline \multicolumn{4}{|l|}{$\mathrm{CRP}, \mathrm{mg} / \mathrm{L}$} \\
\hline SB & 1.19 & 0.65 & .07 \\
\hline LIPA & -0.86 & 0.59 & .14 \\
\hline MVPA & -1.18 & 0.58 & .04 \\
\hline \multicolumn{4}{|l|}{ HOMA-IR } \\
\hline SB & 1.67 & 0.62 & $<.01$ \\
\hline LIPA & -1.10 & 0.56 & .05 \\
\hline MVPA & -2.14 & 0.55 & $<.001$ \\
\hline \multicolumn{4}{|l|}{ Glucose, mg/dL } \\
\hline $\mathrm{SB}$ & 0.48 & 0.65 & .46 \\
\hline LIPA & -0.13 & 0.59 & .83 \\
\hline MVPA & -1.43 & 0.58 & .01 \\
\hline \multicolumn{4}{|l|}{ Sex hormone-binding globulin, $\mathrm{nmol} / \mathrm{L}$} \\
\hline SB & -0.67 & 0.62 & .28 \\
\hline LIPA & 0.31 & 0.56 & .58 \\
\hline MVPA & 1.45 & 0.55 & $<.01$ \\
\hline \multicolumn{4}{|l|}{ Bioavailable estrodial, $\mathrm{pg} / \mathrm{mL}$} \\
\hline SB & 0.85 & 0.48 & .07 \\
\hline LIPA & -0.63 & 0.43 & .15 \\
\hline MVPA & -0.81 & 0.43 & .06 \\
\hline \multicolumn{4}{|l|}{ Testosterone, ng/dL } \\
\hline SB & 0.86 & 0.64 & .17 \\
\hline LIPA & -0.76 & 0.58 & .19 \\
\hline MVPA & -0.27 & 0.57 & .63 \\
\hline
\end{tabular}

Abbreviations: CRP, C-reactive protein; HOMA-IR, homeostatic model assessment of insulin resistance; LIPA, light-intensity physical activity; MVPA, moderate to vigorous physical activity; SB, sedentary behavior. Note: Models adjusted for wear time, age, race, education, smoking status, alcohol consumption, age at diagnosis, cancer stage, aromatase inhibitor use, high blood pressure, and high cholesterol.

Note: Bold values indicate significance at the 0.05 level.

${ }^{a}$ Beta coefficients are log transformed; beta coefficients and SEs reflect associations for $10 \mathrm{~min}$ of activity.

Reallocating 18 minutes of SB to MVPA was beneficial across almost all of the biomarkers, including lower values of insulin $(-9.3 \%$; 95\% CI, -14.2 to -4.1$)$, HOMA-IR (-10.8\%; $95 \% \mathrm{CI},-16.1$ to -5.2$)$, glucose $(-1.7 \%$; $95 \% \mathrm{CI},-3.0$ to -0.4$)$, and higher values of SHBG (7.7\%; 95\% CI, 1.8 to 14.0). The reallocation of SB to MVPA was also associated with lower CRP, but the association was not significant $(-11.3 \%$; 95\% CI, -22.0 to 0.7 ).

Reallocating 18 minutes of LIPA to MVPA also was shown to have beneficial associations, including significantly lower values of insulin $(-9.9 \%$; 95\% CI, -15.5 to -3.9$)$, HOMA-IR (-9.9\%; 95\% CI, -15.5 to -3.9$)$, glucose $(-1.7 \%$; $95 \% \mathrm{CI},-3.1$ to -0.3$)$, and higher values of SHBG (7.6\%; 95\% CI, 1.4 to 14.2). Again, the reallocation of LIPA to MVPA was associated with lower values of CRP, but the association was not significant $(-9.7 \%$; $95 \% \mathrm{CI},-21.2$ to 3.3$)$.

\section{Discussion}

Previous research of daytime activity among breast cancer survivors has focused on the protective effect of MVPA and LIPA, or the deleterious effect of SB independently. In this study, we leveraged objective accelerometer data in a cohort of postmenopausal breast cancer survivors to examine the entirety of waking day activity, taking into consideration the interdependence of these activities. We used isotemporal substitution modeling to explore how the reallocation of time spent in daytime activities is associated with cancer recurrence biomarkers. The results of our isotemporal models suggest that the reallocation of time spent in SB or LIPA to MVPA was associated to significantly lower values of insulin, HOMA-IR, and glucose, and higher values of SHBG. This research adds to the accumulating evidence that suggests that increasing minutes of daily MVPA may have a beneficial effect on cancer recurrence biomarkers.

The results of our study provide insight on the daytime activity profiles of postmenopausal breast cancer survivors. Consistent with previous studies, our results indicate that this population may accumulate daytime activity differently than cancer-free populations. ${ }^{41}$ With accelerometer-measured daytime activity in a highly compliant cohort, our study makes a contribution to the existing evidence. Study participants recorded just under 20 minutes of MVPA a day, and only one-third of the women in our sample are meeting current PA guidelines (150 min/wk of MVPA). Furthermore, participants in our sample accumulated over 5 hours of LIPA (36\% of day), and over 8 hours of SB ( $61 \%$ of day) throughout the day. Thus, our results provide support for the consideration of the amount of time spent in SB and LIPA when developing lifestyle interventions for survivors. ${ }^{42,43}$

Meta-analyses and reviews have concluded that breast cancer survivors who participate in higher levels of PA have significantly lower risk of cancer recurrence. ${ }^{44,45}$ In our study, accelerometermeasured MVPA was significantly associated with lower values of several cancer recurrence biomarkers. In our isotemporal models, increasing minutes of MVPA by reallocating time from SB or LIPA was associated with a greater estimated percentage change in insulin, HOMA-IR, glucose, and SHBG than reallocating minutes of SB to LIPA. These isotemporal results are consistent with findings from previous studies among breast cancer survivors that reported estimated benefits in reallocating time to MVPA. ${ }^{29,30}$ In a sample of 256 breast cancer survivors, Boyle et $\mathrm{al}^{30}$ found a 30-minute reallocation of SB or LIPA to MVPA was associated with lower self-reported waist circumference and BMI. Current lifestyle treatment plans for breast cancer survivors encourage achieving 30 minutes of daily PA and meeting weekly PA guidelines $(150 \text { min of MVPA a week })^{5}$; however, our findings add to the literature by demonstrating that even an 18-minute shift, or $1 \mathrm{SD}$, from SB or LIPA to MVPA may have beneficial associations for breast cancer survivors.

Similar to previous findings, ${ }^{41}$ breast cancer survivors in our cohort spent a large amount of their day sedentary. Both the single variable and isotemporal model results in our analysis suggest decreasing time spent in SB may be beneficial for breast cancer survivors. It is important to note that our results demonstrate significant benefits of decreasing time in SB when it reallocated to MVPA. Although beneficial trends were observed when time was shifted to LIPA, the associations were not significant. Future research is needed to explore the effectiveness and feasibility of targeting SB reduction in breast cancer survivors. In our previous work, we found that increasing sit-to-stand transitions is a feasible intervention target for breast cancer survivors ${ }^{25}$; however, more 


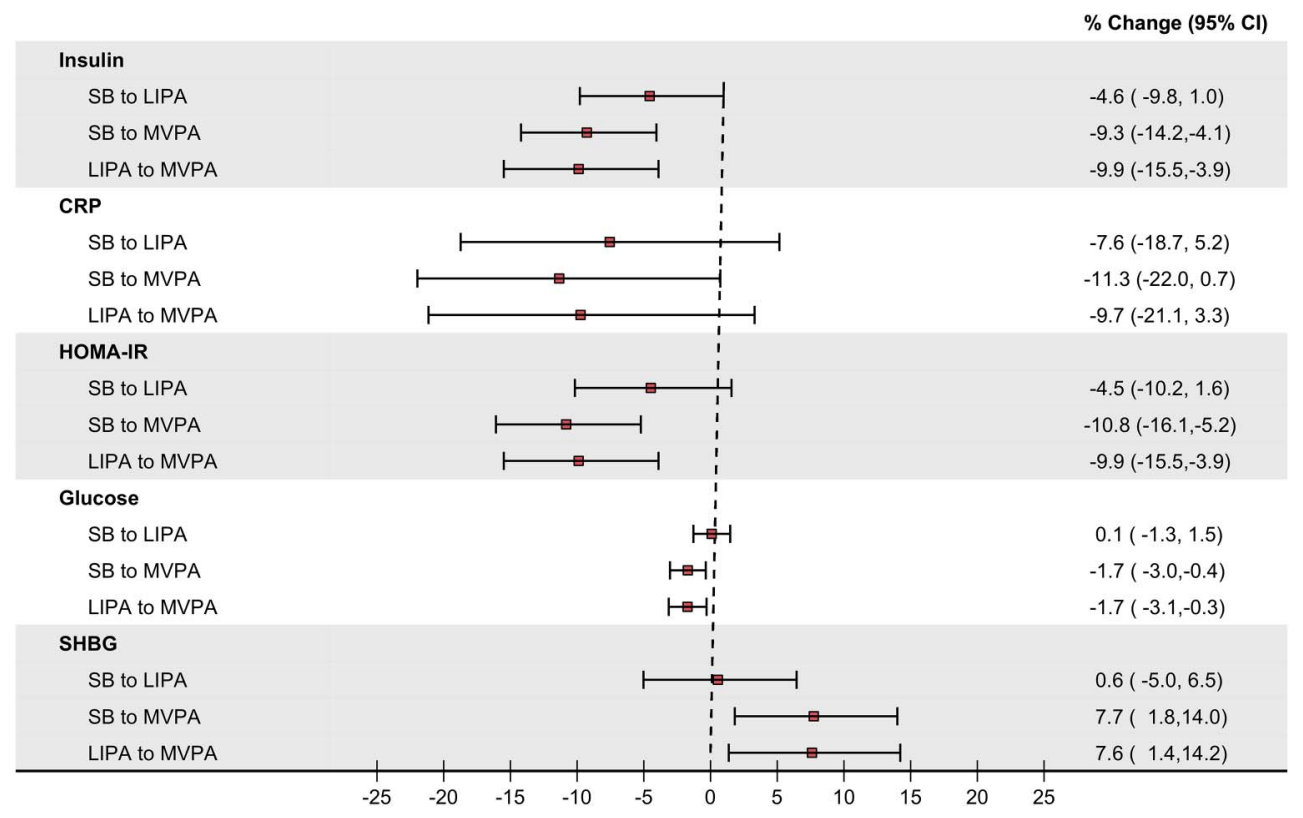

Figure 2 - Reallocation of 1 SD of daytime activity and estimated percentage change to cancer recurrence biomarkers. ${ }^{+}$Models adjusted for wear time, age, race, education, smoking status, alcohol consumption, age at diagnosis, cancer stage, aromatase inhibitor use, high blood pressure, and high cholesterol. CI indicates confidence interval; CRP, C-reactive protein; HOMA-IR, homeostatic model assessment of insulin resistance; LIPA, light physical activity; MVPA, moderate to vigorous physical activity; SB, sedentary behavior; SHBG, sex hormone-binding globulin.

research is needed to test long-term interventions and understand how effects on health outcomes may vary depending on where the time previously spent in SB is shifted to MVPA, LIPA, or sleep.

This analysis has several strengths and limitations. The objective assessment of daytime activity (4-7 d period) using accelerometry is a strength. The use of cross-sectional data, however, only allows for cross-sectional isotemporal models that do not address the real-world difficulties of increasing or decreasing time spent in activity. In addition, although SB, LIPA, and MVPA were measured objectively, accelerometers that were only worn during waking hours and sleep could not be included in the analysis. As research including 24-hour device monitoring increases, ${ }^{46}$ the opportunity to include objectively measured sleep in isotemporal models using 24-hour accelerometer data will increase. In addition, our activities were classified with previously validated and accepted cut points ${ }^{38,47}$; however, these cut points may not be the best way to classify activity behaviors in older populations. ${ }^{48}$ Finally, our sample was limited to overweight or obese breast cancer survivors for the purpose of a weight loss intervention, and therefore, our results may not be generalizable to the entire population of breast cancer survivors.

With an increasing number of breast cancer survivors, it is critical to develop lifestyle treatment guidelines that help this population minimize the risk of cancer recurrence. However, for survivors, public health efforts should not only be focused on staying cancer free, but also on healthy aging, free of all chronic conditions. In this study, multiple biomarkers were examined that are not only cancer recurrence biomarkers, but also markers of cardiometabolic health. Beyond encouraging survivors to increase MVPA, there is a need for a better understanding of how survivors accumulate activity throughout the day and how the interdependence of time spent in daytime activities may be associated to risk of cancer recurrence, as well as overall health. Future studies are needed to examine the interdependence of SB, LIPA, and MVPA in the breast cancer survivor population to inform lifestyle treatment guidelines and the development of survivorship interventions to improve healthy aging.

\section{Acknowledgments}

This work was supported by funding from the National Cancer Institute (U54 CA155435-01). K.M.F. was supported by the National, Heart, Lung and Blood Institute of the National Institutes of Health under the award number T32 HL07779. S.J.H. was supported under the award number K07CA181323.

\section{References}

1. DeSantis C, Ma J, Bryan L, Jemal A. Breast cancer statistics, 2013. CA Cancer J Clin. 2014;64(1):52-62. PubMed ID: 24114568 doi:10. 3322/caac. 21203

2. Miller KD, Siegel RL, Lin CC, et al. Cancer treatment and survivorship statistics, 2016. CA Cancer J Clin. 2016;66(4):271-289. PubMed ID: 27253694 doi:10.3322/caac.21349

3. Siegel RL, Miller KD, Jemal A. Cancer statistics, 2017. CA Cancer J Clin. 2017;67(1):7-30. PubMed ID: 28055103 doi:10.3322/caac. 21387

4. DeSantis CE, Lin CC, Mariotto AB, et al. Cancer treatment and survivorship statistics, 2014. CA Cancer J Clin. 2014;64(4):252-271. PubMed ID: 24890451 doi:10.3322/caac.21235

5. Runowicz CD, Leach CR, Henry NL, et al. American Cancer Society/ American Society of Clinical Oncology Breast Cancer Survivorship Care guideline. CA Cancer J Clin. 2016;66(1):43-73. PubMed ID: 26641959 doi:10.3322/caac.21319

6. Renehan AG, Roberts DL, Dive C. Obesity and cancer: pathophysiological and biological mechanisms. Arch Physiol Biochem. 2008;114(1):71-83. PubMed ID: 18465361 doi:0.1080/13813450 801954303 
7. Mantovani A, Allavena P, Sica A, Balkwill F. Cancer-related inflammation. Nature. 2008;454(7203):436-444. PubMed ID: 18650914 doi:10.1038/nature07205

8. Becker S, Dossus L, Kaaks R. Obesity related hyperinsulinaemia and hyperglycaemia and cancer development. Arch Physiol Biochem. 2009;115(2):86-96. PubMed ID: 19485704 doi:10.1080/ 13813450902878054

9. Roberts DL, Dive C, Renehan AG. Biological mechanisms linking obesity and cancer risk: new perspectives. Annu Rev Med. 2010; 61(1):301-316. doi:10.1146/annurev.med.080708.082713

10. Renehan AG, Frystyk J, Flyvbjerg A. Obesity and cancer risk: the role of the insulin-IGF axis. Trends Endocrinol Metab. 2006;17(8):328336. PubMed ID: 16956771 doi:10.1016/J.TEM.2006.08.006

11. Ollberding NJ, Kim Y, Shvetsov YB, et al. Prediagnostic leptin, adiponectin, c-reactive protein, and the risk of postmenopausal breast cancer. Cancer Prev Res. 2013;6(3):188-195. doi:10.1158/19406207.CAPR-12-0374

12. Key T, Appleby P, Barnes I, Reeves G; Endogenous Hormones and Breast Cancer Collaborative Group. Endogenous sex hormones and breast cancer in postmenopausal women: reanalysis of nine prospective studies. J Natl Cancer Inst. 2002;94(8):606-616. PubMed ID: 11959894 doi:10.1093/jnci/94.8.606

13. Goodwin PJ, Ennis M, Pritchard KI, et al. Fasting insulin and outcome in early-stage breast cancer: results of a prospective cohort study. J Clin Oncol. 2002;20(1):42-51. doi:10.1200/JCO.2002.20. 1.42 .

14. Dossus L, Jimenez-Corona A, Romieu I, et al. C-reactive protein and postmenopausal breast cancer risk: results from the $\mathrm{E} 3 \mathrm{~N}$ cohort study. Cancer Causes Control. 2014;25(4):533-539. PubMed ID: 24504436 doi:10.1007/s10552-014-0355-9

15. Erickson K, Patterson RE, Flatt SW, et al. Clinically defined type 2 diabetes mellitus and prognosis in early-stage breast cancer. J Clin Oncol. 2011;29(1):54-60. PubMed ID: 21115861 doi:10.1200/JCO. 2010.29.3183

16. Islami F, Goding Sauer A, Miller KD, et al. Proportion and number of cancer cases and deaths attributable to potentially modifiable risk factors in the United States. CA Cancer J Clin. 2018;68(1):31-54. PubMed ID: 29160902 doi:10.3322/caac. 21440

17. Kerr J, Anderson C, Lippman SM. Physical activity, sedentary behaviour, diet, and cancer: an update and emerging new evidence. Lancet Oncol. 2017;18(8):e457-e471. PubMed ID: 28759385 doi:10. 1016/S1470-2045(17)30411-4

18. Ibrahim EM, Al-Homaidh A. Physical activity and survival after breast cancer diagnosis: meta-analysis of published studies. Med Oncol. 2011;28(3):753-765. PubMed ID: 20411366 doi:10.1007/ s12032-010-9536-x

19. Beasley JM, Kwan ML, Chen WY, et al. Meeting the physical activity guidelines and survival after breast cancer: findings from the after breast cancer pooling project. Breast Cancer Res Treat. 2012; 131(2):637-643. PubMed ID: 21935600 doi:10.1007/s10549-0111770-1

20. Schmitz KH, Courneya KS, Matthews C, et al. American College of Sports Medicine roundtable on exercise guidelines for cancer survivors. Med Sci Sports Exerc. 2010;42(7):1409-1426. PubMed ID: 20559064 doi:10.1249/MSS.0b013e3181e0c112

21. Groen WG, Van Harten WH, Vallance JK. Systematic review and meta-analysis of distance-based physical activity interventions for cancer survivors (2013-2018): we still haven't found what we're looking for. Cancer Treat Rev. 2018;69:188-203. PubMed ID: 30077954 doi:10.1016/j.ctrv.2018.07.012

22. Thyfault JP, Du M, Kraus WE, Levine JA, Booth FW. Physiology of sedentary behavior and its relationship to health outcomes. Med Sci
Sports Exerc. 2015;47(6):1301-1305. PubMed ID: 25222820 doi:10. 1249/MSS.0000000000000518

23. Lynch BM. Sedentary behavior and cancer: a systematic review of the literature and proposed biological mechanisms. Cancer Epidemiol Biomarkers Prev. 2010;19(11):2691-2709. PubMed ID: 20833969 doi:10.1158/1055-9965.EPI-10-0815

24. Wilmot EG, Edwardson CL, Achana FA, et al. Sedentary time in adults and the association with diabetes, cardiovascular disease and death: systematic review and meta-analysis. Diabetologia. 2012; 55(11):2895-2905. PubMed ID: 22890825 doi:10.1007/s00125-0122677-z

25. Hartman SJ, Marinac CR, Cadmus-Bertram L, et al. Sedentary behaviors and biomarkers among breast cancer survivors. $J$ Phys Act Health. 2018;15(1):1-6. doi:10.1123/jpah.2017-0045

26. Füzéki E, Engeroff T, Banzer W. Health benefits of light-intensity physical activity: a systematic review of accelerometer data of the National Health and Nutrition Examination Survey (NHANES). Sports Med. 2017;47(9):1769-1793. doi:10.1007/s40279-0170724-0

27. Healy GN, Dunstan DW, Salmon J, et al. Objectively measured lightintensity physical activity is independently associated with 2-h plasma glucose. Diabetes Care. 2007;30(6):1384-1389. PubMed ID: 17473059 doi:10.2337/dc07-0114

28. Mekary RA, Willett WC, Hu FB, Ding EL. Isotemporal substitution paradigm for physical activity epidemiology and weight change. Am J Epidemiol. 2009;170(4):519-527. PubMed ID: 19584129 doi:10. 1093/aje/kwp163

29. Ehlers DK, Fanning J, Salerno EA, et al. Replacing sedentary time with physical activity or sleep: effects on cancer-related cognitive impairment in breast cancer survivors. BMC Cancer. 2018;18(1):685. PubMed ID: 29940894 doi:10.1186/s12885-018-4603-3

30. Boyle T, Vallance JK, Buman MP, Lynch BM. Reallocating time to sleep, sedentary time, or physical activity: associations with waist circumference and body mass index in breast cancer survivors. Cancer Epidemiol Biomarkers Prev. 2017;26(2):254-260. PubMed ID: 27780817 doi:10.1158/1055-9965.EPI-16-0545

31. Patterson RE, Marinac CR, Natarajan L, et al. Recruitment strategies, design, and participant characteristics in a trial of weight-loss and metformin in breast cancer survivors. Contemp Clin Trials. 2016;47:64-71. PubMed ID: 26706665 doi:10.1016/j.cct.2015. 12.009

32. Choi L, Liu Z, Matthews CE, Buchowski MS. Validation of accelerometer wear and nonwear time classification algorithm. Med Sci Sports Exerc. 2011;43(2):357-364. doi:10.1249/MSS.0b013e31 81ed61a3

33. Buchner DM, Rillamas-Sun E, Di C, et al. Accelerometer-measured moderate to vigorous physical activity and incidence rates of falls in older women. J Am Geriatr Soc. 2017;65(11):2480-2487. PubMed ID: 28755415 doi:10.1111/jgs. 14960

34. Lee I-M, Shiroma EJ, Evenson KR, Kamada M, LaCroix AZ, Buring JE. Using devices to assess physical activity and sedentary behavior in a large cohort study, the women's health study. J Meas Phys Behav. 2018;1(2):60-69. PubMed ID: 30666321 doi:10.1123/jmpb.20180005

35. Marinac CR, Godbole S, Kerr J, et al. Objectively-measured physical activity and cognitive functioning in breast cancer survivors HHS public access. J Cancer Surviv. 2015;9(2):230-238. PubMed ID: 25304986 doi:10.1007/s11764-014-0404-0

36. Hartman SJ, Marinac CR, Bellettiere J, et al. Objectively measured sedentary behavior and quality of life among survivors of early stage breast cancer. Support Care Cancer. 2017;25(8):2495-2503. PubMed ID: 28326507 doi:10.1007/s00520-017-3657-0 
37. LaCroix AZ, Rillamas-Sun E, Buchner D, et al. The objective physical activity and cardiovascular disease health in older women (OPACH) study. BMC Public Health. 2017;17(1):1-12. doi:10.1186/ s12889-017-4065-6

38. Freedson PS, Melanson E, Sirard J. Calibration of the computer science and applications, inc. accelerometer. Med Sci Sports Exerc. 1998;30(5):777-781. PubMed ID: 9588623 doi:10.1097/00005768199805000-00021

39. Patterson RE, Marinac CR, Sears DD, et al. The effects of metformin and weight loss on biomarkers associated with breast cancer outcomes. J Natl Cancer Inst. 2018;110(11):1239-1247. PubMed ID: 29788487 doi:10.1093/jnci/djy040

40. R Core Team. R: a language and environment for statistical computing. 2013. http://www.r-project.org/

41. Phillips SM, Dodd KW, Steeves J, Mcclain J, Alfano CM, Mcauley E. Physical activity and sedentary behavior in breast cancer survivors: new insight into activity patterns and potential intervention targets HHS public access. Gynecol Oncol. 2015;138(2):398-404. PubMed ID: 26026737 doi:10.1016/j.ygyno.2015.05.026

42. Lynch BM, Dunstan DW, Healy GN, Winkler E, Eakin E, Owen N. Objectively measured physical activity and sedentary time of breast cancer survivors, and associations with adiposity: findings from NHANES (2003-2006). Cancer Causes Control. 2010;21:283288. doi:10.2307/25621357
43. Lynch BM, Dunstan DW, Vallance JK, Owen N. Don't take cancer sitting down. Cancer. 2013;119(11):1928-1935. PubMed ID: 23504979 doi:10.1002/cncr.28028

44. Cormie P, Zopf EM, Zhang X, Schmitz KH. The impact of exercise on cancer mortality, recurrence, and treatment-related adverse effects. Epidemiol Rev. 2017;39(1):71-92. PubMed ID: 28453622 doi:10. 1093/epirev/mxx007

45. Lahart IM, Metsios GS, Nevill AM, Carmichael AR. Physical activity, risk of death and recurrence in breast cancer survivors: a systematic review and meta-analysis of epidemiological studies. Acta Oncol. 2015;54(5):635-654. doi:10.3109/0284186X.2014.998275

46. Rosenberger ME, Buman MP, Haskell WL, McConnell MV, Carstensen LL. Twenty-four hours of sleep, sedentary behavior, and physical activity with nine wearable devices. Med Sci Sports Exerc. 2016;48(3):457-465. PubMed ID: 26484953 doi:10.1249/ MSS.0000000000000778

47. Matthews CE, Chen KY, Freedson PS, et al. Amount of time spent in sedentary behaviors in the United States, 2003-2004. Am J Epidemiol. 2008;167(7):875-881. PubMed ID: 18303006 doi:10.1093/aje/ kwm390

48. Kerr J, Marshall SJ, Godbole S, et al. Using the SenseCam to improve classifications of sedentary behavior in free-living settings. Am J Prev Med. 2013;44(3):290-296. PubMed ID: 23415127 doi:10.1016/j. amepre.2012.11.004 